\title{
Comparing Transformation Possibilities of Topological Functioning Model and BPMN in the Context of Model Driven Architecture
}

\author{
Arturs Solomencevs \\ Department of Applied Computer Science, Riga Technical University, Latvia
}

\begin{abstract}
The approach called "Topological Functioning Model for Software Engineering" (TFM4SE) applies the Topological Functioning Model (TFM) for modelling the business system in the context of Model Driven Architecture. TFM is a mathematically formal computation independent model (CIM). TFM4SE is compared to an approach that uses BPMN as a CIM. The comparison focuses on CIM modelling and on transformation to UML Sequence diagram on the platform independent (PIM) level. The results show the advantages and drawbacks the formalism of TFM brings into the development.
\end{abstract}

Keywords - CIM to PIM transformation, formal CIM, model driven architecture, topological functioning model.

\section{INTRODUCTION}

OMG's Model Driven Architecture (MDA) is a set of guidelines for system development, which pioneers application of models. MDA separates the viewpoints on the information system - it suggests three viewpoints with corresponding models: a computation independent model (CIM) focuses on information system's environment and is used to specify requirements; platform independent model (PIM) focuses on the information system but hides platform specific details; and platform specific model (PSM) [1]. Model transformation is considered to be an important part of MDA. To get the source code, we need to go by the path: CIM $\rightarrow$ $\mathrm{PIM} \rightarrow \mathrm{PSM} \rightarrow$ source code [1].

There is a belief that it is essential to start software development with modelling the business system, or in other words with modelling the environment of the planned information system [2], [3], [4]. Understanding of how the information system will interact with the business system leads to an appropriate design. Thus, CIM needs to be created at the beginning of the development process - this assertion is the basis of this article.

The problem domain is the part of the world in which the software is required to bring about some effect desired by the customer [2]. The solution domain is a system (e.g., business system), which is supported by the planned information system. Both problem domain and solution domain can be specified by CIM [5]. The solution domain CIM must conform to the problem domain CIM. It is possible to transform the solution domain CIM to PIM level design models [6].

There is a shortcoming in MDA guide [1]. OMG says nothing essential about the computation independent view and accordingly about the CIM. The weakness of MDA is that there is nothing well formalised and/or transformable at the beginning of the software development life cycle [3].

Our group works on dealing with the mentioned issue and has developed an approach called "Topological Functioning Model for Software Engineering" (TFM4SE). This approach uses the Topological Functioning Model (TFM) as a formal CIM.

As prof. J. Osis states in [7], "TFM is a formal model which describes the functioning of a system. TFM has a solid mathematical base. It is represented in a form of a topological space $(X, \Theta)$, where $X$ is a finite set of functional features of the system under consideration, and $\Theta$ is topology that satisfies axioms of topological structures and is represented in a form of a directed graph. The TFM's functional features describe the system's physical or biological characteristics that are relevant for the normal functioning of the system. The TFM's topology consists of cause-effect relations between functional features. Cause-effect relation means that appearance of one functional feature causes an appearance of the other one. Causal chains must form at least one functioning cycle within TFM." All formal definitions of TFM elements are given in [8].

In the author's opinion, the improvement of the results of object-oriented system analysis and modelling lays in using formal methods. Formal approaches allow defining formal or semi-formal model transformations (including reverse engineering [9]), which are the key part of MDA, and formal tracing of modelling artefacts. Furthermore, the power of traditional engineering is that engineers do trust in theory, mathematics and formal methods [3], and this power can be used in software development. TFM4SE is a formal method, which has a mathematical model - TFM - in its core.

The goal of the current research is to compare TFM4SE with other model-driven approaches that suggest creating CIM level models which can be transformed to PIM. For this paper, the author narrows the scope and reviews approaches that use BPMN models [10] on the CIM level. BPMN is widely used in software development for business modelling. This notation is easy to work with and is understandable for business people and software developers (training is required to freely use it). BPMN does not have mathematical basis. Therefore, the author is interested in comparing CIM-TFM to CIM-BPMN, and finding out the benefits and drawbacks caused by the 
formalism of TFM comparing to informal BPMN business model in the field of software development.

The paper is structured as follows. In Section 2 approaches that provide $\mathrm{CIM} \rightarrow$ PIM transformation discovered during the research are briefly reviewed; also the major opportunities of TFM4SE are represented. In Section 3 the comparison of TFM4SE with one of the CIM-BPMN approaches on the basis of an example is made. In Section 4 conclusions about the research are given.

\section{RELATED STUDIES}

There are a number of software development approaches that use BPMN for CIM modelling and provide CIM $\rightarrow$ PIM transformation. The approach described in [11] applies BPMN collaboration and business process diagrams on the CIM level, and defines rules for transformation to UML Class diagram and Use case diagram on the PIM level. Service-oriented approach [12] proposes transforming BPMN models into SoaML model [13] and into UML Component model. Method of [14] is also service-oriented and uses SoaML for PIM modelling. Another service-oriented approach - [15] - applies the use case model and activity diagram on the PIM level. Research of [16] focuses on security requirements and models security along with business processes. Approach [17] provides ways of getting class diagram and sequence diagrams from BPMN business process models.

Not only BPMN is applied to CIM modelling. An approach represented in [18] applies features as the elements of CIM, and components - as elements of PIM. Paper [19] proposes automatically generating an activity diagram from a use case description. Data warehouse development method [20] uses a UML profile for the $i^{*}$ modelling framework [21] for CIM modelling, and provides transition to a conceptual model on the PIM level. Research of [22] proposes using UML activity diagram for business process modelling and for defining the requirements, and also for transforming CIM into PIM. Data flow diagrams are used on the CIM level by [23] approach, which also provides the obtaining of PIM models.

Finally, TFM4SE approach in brief. The approach provides ways of obtaining TFM from the knowledge about a business system [24]-[27]; derivation of use case diagrams from TFM [28]; transformations of TFM to the most popular UML [29] design models on PIM level, e.g., Class diagram, Sequence diagram, Communication diagram [30]-[32]; formalizing tracing links between modelling artefacts [33]; and other opportunities for formalized software development.

\section{COMPARISON OF TFM4SE WITH CIM-BPMN APPROACH ON THE BASIS OF AN EXAMPLE}

In the previous section, the author has reviewed approaches that use BPMN in CIM modelling and provide CIM $\rightarrow$ PIM transformation. The author finds that the approach represented in [17] is the most well-elaborated, because the article is deep and of good quality. Also, the approach is wide enough that its parts are covered by other articles: [34]-[37]. That is why this approach has been chosen to compare with TFM4SE. For convenience, from now on this approach is called "CIMBPMN approach".

In CIM-BPMN approach, there are the following models on the CIM level: business process models (BPM) that are based on BPMN and a business use case model. High-level BPMs are distinguished from low-level BPMs. High-level models are more abstract and contain collapsed sub-processes. Low-level models describe in detail the expanded sub-processes from the high-level models. It is written in [17] that "PIM level contains three models: Domain class diagram; Business rules; and Sequence diagram of system's external behaviour." Domain class diagram is a UML Class diagram with attributes and relations, but without methods. Business rules focus on structural assertions and define structure, relationships and integrity constraints on data. "Sequence diagram of system's external behavior is a UML Sequence diagram that shows interactions between actors and the whole system as unique entity" [17]. The approach also proposes a way to obtain the "Sequence diagram of system's internal behaviour" [36]. However, this transformation requires developing additional descriptions.

CIM-BPMN approach provides construction of the following UML models from BPMN business model: use case diagram; sequence diagram; and class diagram. TFM4SE also supports obtaining of these models from TFM. However, it is not possible to review the creation of all the mentioned models in one article. Therefore, the scope is narrowed and the paper focuses on CIM modelling, and on transformation to PIM sequence diagram. The article of CIM-BPMN [17] represents an example (a case study) of BPMN modelling and of acquiring the mentioned models. This example is taken as the basis for the comparison.

\section{A. Verbal Description of the System}

Verbal description is given in [17]. It is a description of an e-commerce web site. It describes a solution domain (system "to-be"), i.e., a business system that is supported by the planned information system. Normally, a problem domain is described, i.e., a business system that has not been supported by the planned information system yet. However, e-commerce specific feature is that there is no business without the information system; hence, there is no problem domain. By studying BPMN models of the example from the article, the author has concluded that the verbal description is not complete. The author has refined the description so that it corresponds to the mentioned BPMN models. Comprehensive verbal description is needed for creation of TFM. The description is given below.

Designations used in the description are the following. Italic - nouns, real world objects and their attributes. Bold verbs and conditions that define the appropriate actions.

Any web surfer can access the web site and search for product of different categories (Book, informatics....) and collect them in web surfer's cart. Web surfer can manage this cart at any time to add/remove products or to change the quantity of product. When web surfer is convinced, web surfer can check out the order and pay for the order that will 
be shipped (delivered) to web surfer's shipping address. Web surfer must login with web surfer's account or register a new account if web surfer's does not have an account for the web site.

When clerk receives the payment, he prepares order for shipping. Web surfer can check the order status and review the order. Clerk sends the prepared order, and delivery company delivers the order to web surfer's shipping address. Finally, the web surfer receives his order.

Web surfer can leave the web site.

Order checkout expansion. All products in the web surfer's cart are shown to the web surfer. Web surfer validates the cart, and if he is not satisfied, he cancels the order. Otherwise, the web surfer fills in customer information. Web surfer fills in his shipping address. The information system checks whether web surfer's shipping address is deliverable. If web surfer's shipping address is not deliverable, web surfer is asked to fill in another shipping address, and information system checks it the same way. If the web surfer's shipping address is deliverable, then web surfer fills in web surfer's billing address. Web surfer selects shipping mode. Information system checks whether shipping mode is available for web surfer's shipping address; if it is not available, web surfer is asked to select another shipping mode, and information system checks it the same way. If the shipping mode is available for web surfer's shipping address, then web surfer validates the order. If order validation is unsuccessful, web surfer cancels the order. If order validation is successful, web surfer pays for the order (Payment sub-process is expanded later). Information system registers order as a paid order, and notifies clerk about the new paid order.

Payment expansion. Web surfer fills in his credit card information. Web surfer validates his billing address, then validates his shipping address, then confirms payment. Banking system checks whether web surfer's credit card is valid. If web surfer's credit card is not valid, the banking system rejects the payment. Otherwise, the banking system makes the payment transaction and saves it, and notifies web surfer about the successful payment transaction.

\section{B. Functional Features and High-Level Business Models}

According to the formal method of TFM construction [24], in order to define functional features, verbs denoting actions, their preconditions and business rules are to be found in informal description of the system. Preconditions specify a set of conditions that allow triggering a functional feature. A business rule usually prevents, provokes or allows triggering certain processes and defines or constrains some business process aspects. Each action, precondition or business rule either has to introduce a new appropriate functional feature or it should be attached to the already defined one. Besides, entities that are responsible for performing an action of the functional feature are defined. "Functionality can be subordinated to the system under consideration (inner) or to other systems (external)" [24].

Functional feature is expressed in the following form [24]:

"<action>-ing the <result> [to, into, in, by, of, from] $a(n)$

$$
\text { <object>" }
$$

e.g., Adding the product to a cart.

Object gets the result of the action. The obtained list of TFM's functional features is given in Table I.

TABLE I

Functional Features of E-COMmerce Web Shop System

\begin{tabular}{|l|l|l|l|l|}
\hline ID & \multicolumn{1}{|c|}{ Functional feature } & \multicolumn{1}{|c|}{ Preconditions } & \multicolumn{1}{c|}{ Entity } & Inner / External \\
\hline 1 & Accessing a web site & & web surfer & external \\
\hline 2 & Searching for a product & & web surfer & inner \\
\hline 3 & Adding the product to a cart & & web surfer & inner \\
\hline 4 & Managing a cart & & web surfer & inner \\
\hline 5 & Removing the product from a cart & web surfer & inner \\
\hline 6 & Changing the quantity of a product & & web surfer & inner \\
\hline 7 & Checking out an order & [when web surfer is convinced] & web surfer & inner \\
\hline 8 & Paying for an order & [if order validation is successful] & web surfer & inner \\
\hline 9 & $\begin{array}{l}\text { Delivering the order to a web surfer's shipping } \\
\text { address }\end{array}$ & & delivery company & external \\
\hline 10 & Logging in with a web surfer's account & [if web surfer has an account] & web surfer & inner \\
\hline 11 & Registering a new web surfer's account & [if web surfer does NOT have an account] & web surfer & inner \\
\hline 12 & Preparing for shipping an order & [when clerk is notified about receiving the payment] & clerk & inner \\
\hline 13 & Checking the status of an order & & web surfer & external \\
\hline 14 & $\begin{array}{l}\text { Sending the prepared order to a delivery } \\
\text { company }\end{array}$ & [when order is prepared for shipping] & clerk & inner \\
\hline 15 & Receiving an order & & web surfer \\
\hline 16 & Showing the contents of cart to a web surfer & & information system & inner \\
\hline 17 & Validating a cart & & web surfer & inner \\
\hline
\end{tabular}




\begin{tabular}{|c|c|c|c|c|}
\hline ID & Functional feature & Preconditions & Entity & Inner / External \\
\hline 18 & Cancelling an order & $\begin{array}{l}\text { [if web surfer is NOT satisfied with the contents of } \\
\text { the cart] }\end{array}$ & web surfer & inner \\
\hline 19 & Filling in customer information & $\begin{array}{l}\text { [if web surfer is satisfied with the contents of the } \\
\text { cart] }\end{array}$ & web surfer & inner \\
\hline 20 & Filling in a shipping address & & web surfer & inner \\
\hline 21 & Checking a shipping address & & information system & inner \\
\hline 22 & Asking to fill in another shipping address & $\begin{array}{l}\text { [if web surfer's shipping address is NOT } \\
\text { deliverable] }\end{array}$ & information system & inner \\
\hline 23 & Filling in a billing address & [if web surfer's shipping address is deliverable] & web surfer & inner \\
\hline 24 & Selecting the shipping mode of an order & & web surfer & inner \\
\hline 25 & Checking a shipping mode of an order & & information system & inner \\
\hline 26 & Asking to select another shipping mode & $\begin{array}{l}\text { [if shipping mode is NOT available for web surfer's } \\
\text { shipping address] }\end{array}$ & information system & inner \\
\hline 27 & Validating an order & $\begin{array}{l}\text { [if shipping mode is available for web surfer's } \\
\text { shipping address] }\end{array}$ & web surfer & inner \\
\hline 28 & Cancelling an order & [if order validation is unsuccessful] & web surfer & inner \\
\hline 29 & Registering a paid order & [if payment was successful] & information system & \\
\hline 30 & Notifying clerk about a new paid order & & information system & \\
\hline 31 & Filling in the information of a credit card & & web surfer & inner \\
\hline 32 & Validating a billing address & & web surfer & inner \\
\hline 33 & Validating a shipping address & [if billing address validation is successful] & web surfer & inner \\
\hline 34 & Confirming a payment & [if shipping address validation is successful] & web surfer & inner \\
\hline 35 & Checking a credit card & & banking system & external \\
\hline 36 & Rejecting a payment & [if web surfer's credit card is NOT valid] & banking system & external \\
\hline 37 & Making a payment transaction & [if web surfer's credit card is valid] & banking system & external \\
\hline 38 & Saving a payment transaction & & banking system & external \\
\hline 39 & $\begin{array}{l}\text { Notifying web surfer about a successful } \\
\text { payment transaction }\end{array}$ & & banking system & external \\
\hline 40 & $\begin{array}{l}\text { Notifying information system about a successful } \\
\text { payment transaction }\end{array}$ & & banking system & external \\
\hline 41 & Leaving a web site & & web surfer & external \\
\hline 42 & Reviewing an order & & web surfer & external \\
\hline
\end{tabular}

"After definition of functional features topology $\Theta$ (causeand-effect relationships) between them is introduced" [24]. At first, topological space on a higher abstraction level is constructed (see Fig. 1). It is possible to construct topological space by using TFM editor [38]. However, TFM editor is not used for this paper due to the limit of free space (figures with TFM would be much bigger).

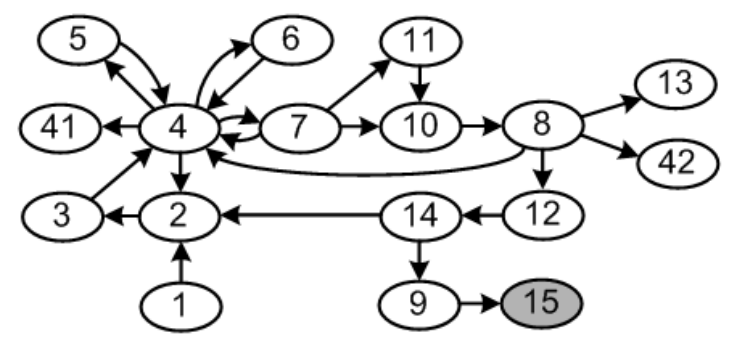

Fig. 1. Topological space of the solution domain on high level of abstraction.

Topological space of the solution domain (Fig. 1) represents both web shop's inner functional features - set $N$, and external functional features - set $M$ [38]. In the case of the example, set
$M=\{1,9,13,15,41,42\}$; set $N=\{2,3,4,5,6,7,8,10,11$, $12,14\}$. "To separate TFM of the system under consideration from the topological space, the closure operation over the set $\mathrm{N}$ is applied" [7], [24]:

$$
X=[N]=\bigcup_{i=1}^{n} X_{i} .
$$

" $X$ is a set of functional features of the system's TFM; $X_{i}$ is an adherence vertex of the set $N$; and $n$ is a number of adherence vertices of $N$, i.e. capacity of $X(1)$. An adherence vertex of the set $N$ is a vertex each neighborhood of which includes at least one vertex from the set $N$ ' [7], [24]. New definition of neighbourhood is introduced, because it positively affects the result of the closure operation. The neighbourhood of a vertex is a subset which contains this vertex and all vertices that are adjacent to it. To perform closure of set $N$, at first, neighbourhood of each vertex of $N$ should be found. For example, in the case study:
(2) $=\{2,1,3,4,14\}$
$(12)=\{12,8,14\}$
(3) $=\{3,2,4\} \ldots$
$(14)=\{14,2,9,12\}$. 


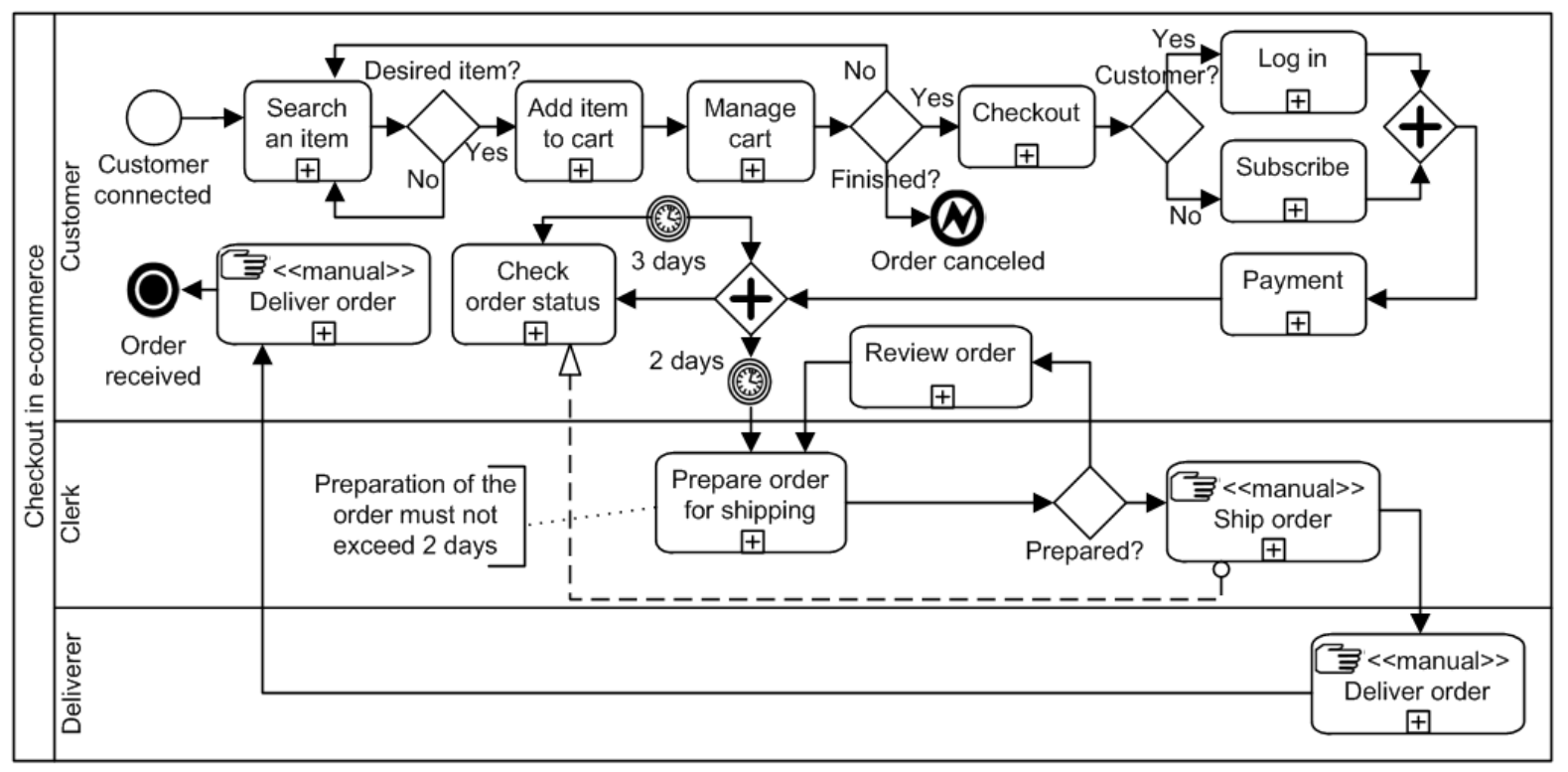

Fig. 2. BPMN business process model on high abstraction level, adapted from [17].

Then, a union of neighbourhoods is obtained. This union is the set $X=[N]=\{1,2,3,4,5,6,7,8,9,10,11,12,13,14,41$, $42\}$. Thus, TFM does not contain functional feature 15 , and this functional feature is considered to be out of bounds of web shop's system.

The set $M$ contains inputs and outputs [24]. Set of inputs = $\{1\}$. Set of outputs $=\{9,13,41,42\}$. In this TFM, the main functional cycle is as follows: 2-3-4-7-10-8-12-14-2 (Fig. 1).

Figure 2 represents the BPMN business process model on high level of abstraction that is given in CIM-BPMN approach case study. This model corresponds to topological space shown in Fig. 1.

The expansion of the following processes is needed: order checkout and payment. To do this, a formal mechanism provided by topological modelling - continuous mapping can be used. As it is stated in [7] and [39], "If some more detailed functioning system is formed by substitution of a subset of specialized functional features for some functional feature, then continuous mapping exists between a detailed model and a simplified parent topological model. In the topological digraph $G^{*}\left(X^{*}, U^{*}\right)$, the direction of arcs, which join the specialized point subset nodes with other nodes, is determined by the direction of the arcs, which join the replaced point with the corresponding nodes of the digraph $\mathrm{G}$ $(X, U)$ ". To put it simply, continuous mapping allows substituting a subset of functional features with a more detailed subset, and vice versa.

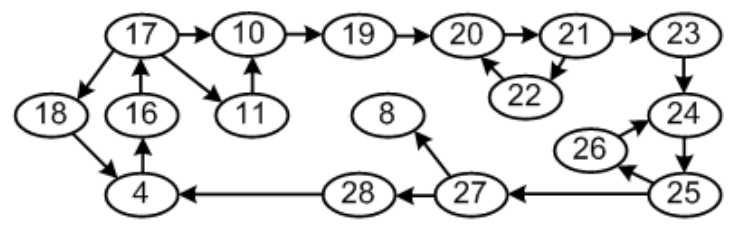

Fig. 3. Topological space part that represents detailed functionality of checkout sub-process.
Figure 3 shows the part of topological space that represents detailed functionality of checkout. This part of TFM has been obtained by applying continuous mapping. A subset of functional features "7 Checking out an order", "10: Logging in with a web surfer's account" and "11: Registering a new web surfer's account" is continuously mapped onto the set of functional features that is represented in Fig. 3. Also, all cause-effect relations with surrounding functional features, i.e., "4: Managing a cart" and "8: Paying for an order", are retained. Since all functional features in Fig. 3 belong to system's inner functional features, they also belong to set $X$ of web shop's TFM. By increasing the level of detail, a new precondition for functional feature was found "8: Paying for an order" - [if order validation is successful].

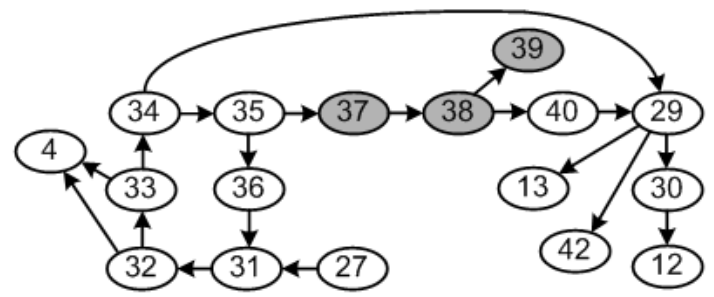

Fig. 4. Topological space part that represents detailed functionality of payment sub-process.

Figure 4 illustrates the part of topological space that represents detailed functionality of payment. Functional feature "8: Paying for an order" is continuously mapped to the represented set. Functional feature " 27 : Validating an order" is used instead of "10: Logging in with a web surfer's account" to conform to the more detailed functionality description of the checkout (see Fig. 3). $X_{\text {Payment }}=\left[N_{\text {Payment }}\right]=\{4,12,13,27$, $29,30,31,32,33,34,35,36,40,42\}$. Thus, TFM does not contain functional features $\{37,38,39\}$. 


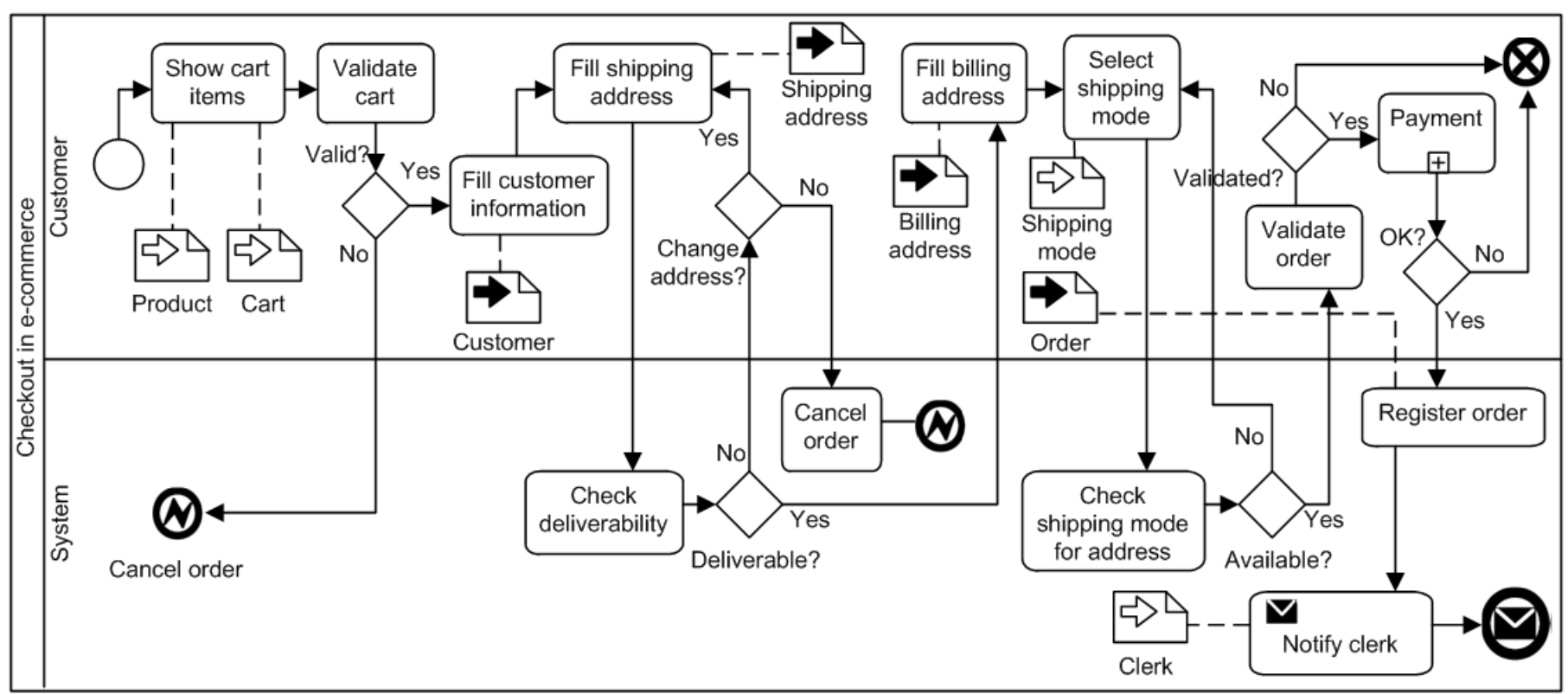

Fig. 5. BPMN model of expanded checkout sub-process, adapted from [17].

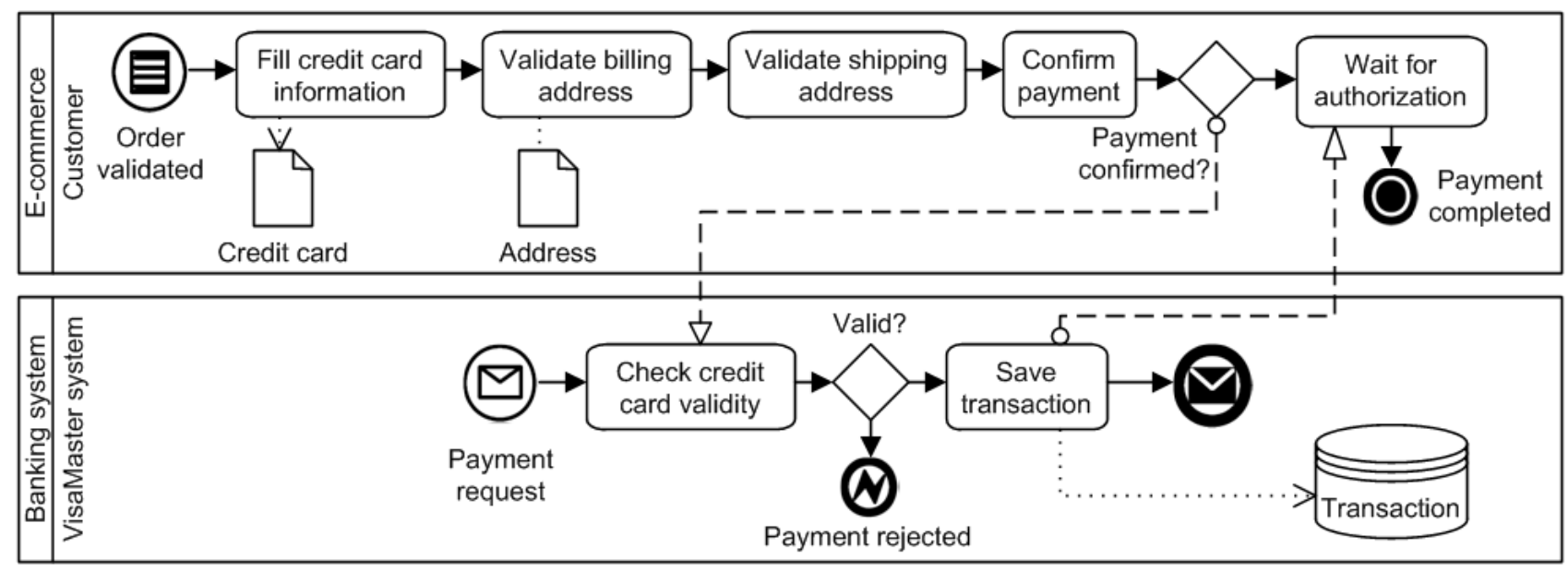

Fig. 6. BPMN model of expanded payment sub-process, adapted from [17].

In CIM-BPMN, more detailed models - low-level BPMs are obtained by expanding collapsed sub-processes of highlevel BPM [17]. Figure 5 displays a BPM of the expanded checkout sub-process, and Figure 6 - of the expanded payment sub-process.

The main difference between the approaches concerning the expansion of sub-processes is the following. In TFM, the formal operation is used - continuous mapping. The new subset must keep all cause-effect relations that the substituted subset had with other functional features in TFM. In BPMN, there is no such a strict rule. Therefore, an ambiguity may arise in BPMN model. Let us consider a case when the payment is rejected by a banking system (payment expansion). Low-level BPM model (see Fig. 6) does not make it clear what should happen after the payment rejection, and highlevel BPM (see Fig. 2) also does not help. In TFM there is no such ambiguity. High-level TFM (see Fig. 1) tells us nothing about payment rejection, so the rejection must be handled by a subset of functional features that substitutes "8: Paying for an order". Figure 1 shows us a cause-effect relation between "8: Paying for an order" and " 4 : Managing a cart", and this relation must be retained in the expanded TFM. After the payment rejection, it makes sense to give web surfer an opportunity to fill credit card information once again, or to go back to cart management. In Figure 4, after "36: Rejecting a payment" an execution goes to "31: Filling in the information of a credit card". Web surfer can refill data or skip this step. During validation of billing and shipping addresses (features 32 and 33) web surfer can go back to "4: Managing a cart". Therefore, processing of payment will be cancelled. Thus, dealing with the payment rejection is explicitly and unambiguously described. It can be seen that formalism of TFM helps to achieve consistency between abstract and detailed models. 


\section{Transformation to PIM Sequence Diagram}

TFM4SE defines the obtaining of UML Sequence diagram from TFM [40]. Each functional feature will be supported by a class method. So for each functional feature architect must come up with the name of a class and the name of a method, which will realize the functional feature [41]. There is no point in assigning classes and methods to functional features that are not realised by the information system. Examples are given in Table II. The rows starting with ID $=29$ are used in construction of sequence diagram later in this section.

TABLE II

EXAMPLES OF ClASS AND METHOD NAMES

\begin{tabular}{|l|l|l|}
\hline ID & Class & Method \\
\hline 1 & Cart & accessWebSite() \\
\hline 2 & ProductSearcher & searchForProduct() \\
\hline 29 & OrderRegistry & registerPaidOrder() \\
\hline 31 & WebSurferAccount & fillInCreditCardInfo() \\
\hline 32 & Order & waitForValidation() \\
\hline 33 & Order & waitForValidation() \\
\hline 34 & Order & confirmPayment() \\
\hline 35 & Order & sendOrderInfoToBank() \\
\hline 36 & Order & rejectPayment() \\
\hline 40 & Order & successfulPayment() \\
\hline
\end{tabular}

In TFM4SE, the scope of sequence diagram is determined by system goal or by functional requirement. For each system goal or requirement one sequence diagram should be developed [40]. For this comparison, the obtaining of one sequence diagram is shown. Thus, a corresponding system goal needs to be defined. Guidelines for identifying system goals are published in [28]. This process consists of the 5 following steps.

Step 1: Identification of business actors. "Actors are external entities interacting directly with the business system that establish business goals within the business system" [28]. Actors are external entities in the TFM, where they can be represented as an external system itself (e.g., a company, a customer) or as an entity that is responsible for initiation of a functional feature from the external environment, or a functional feature that describes system interaction with the external environment [28]. In the case study, by analysing Table I (paying the most attention to columns "Entity" and "Inner/External"), the following business actors were identified: web surfer, delivery company, banking system.

Step 2: Identification of business workers. "Workers are inner entities interacting directly with the business system that either establish system goals or realize some business goals. Workers are people, roles, etc. that work within the business system and can be identified from the TFM" [28]. Again, after analysing Table I, the identified business workers are: clerk and information system.

Step 3: Identification of business goals. Each identified business user (actor or worker) that interacts with the business system has his business goal [28]. The author does not identify business goals for all users, only for the banking system. Its only business goal is to "Make a payment transaction for the order," which has two sub-goals: "Get order and credit card information" and "Make payment transaction or reject payment." Therefore, the banking system needs to get information about order and web surfer's credit card, and then either make payment transaction or reject payment.

Step 4: Associating functionality with business goals. "Each direct goal is to be corresponded to the functional features (one or many) of the system. For each user's goal, an input functional feature (input transaction), an output functional feature (output transaction), and a functional feature chain between them should be identified. Transaction may consist of the only one functional feature" [28]. The author identified the following functional feature chain for getting information about the order and the credit card information: "31-32-33-3435." Here, "31: Filling in the information of a credit card" is considered to be an input functional feature. In its turn, "35: Checking a credit card" is an output functional feature (output data are order and credit card details which are received by the banking system). If the payment is processed successfully, functional features "40: Notifying information system about a successful payment transaction" and "29: Registering a paid order" are executed; otherwise - "36: Rejecting a payment."

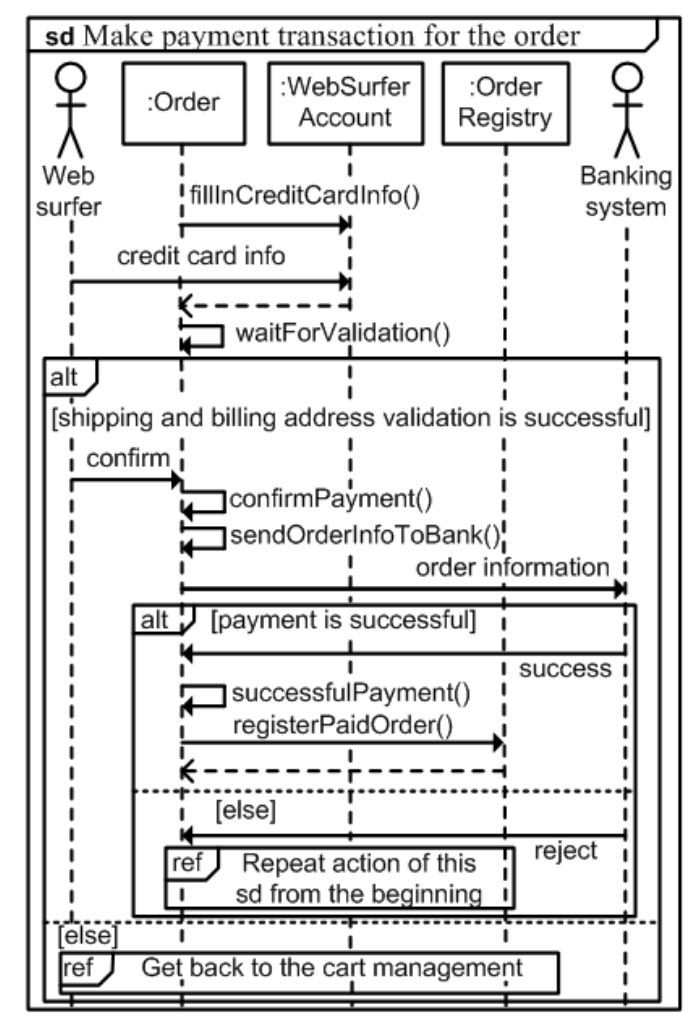

Fig. 7. Sequence diagram of making a payment transaction for the order, obtained by applying TFM4SE.

Step 5: Identification of system goals. "Both actors and workers can be users of the computerized system. Functional features to be implemented that are associated with a business goal constitute functionality that is dedicated to reach a corresponding system goal. Thus, each system goal is mapped to a corresponding business goal" [28]. The author has 
identified the system goal "Make a payment transaction for the order," which maps to the business goal with the same name (defined during the $3^{\text {rd }}$ step). Functional features to be implemented to satisfy this system goal are: $\{29,31,32,33$, $34,35,36,40\}$ (all that were mentioned in the $4^{\text {th }}$ step).

After defining a system goal and identifying the functional features that correspond to it, we can create a sequence diagram for this goal. Vertices of TFM with the same class names (see Table II) should be merged, and, while merging, all relationships between vertices (see Fig. 4) should be kept. The relations from TFM serve as message sending between objects in sequence diagram [40]. Figure 7 shows the resulting sequence diagram. This transformation requires partial participation of the architect, i.e., information exchange between the system and actors needs to be specified, and interaction operators, e.g., alternative and loop, need to be properly described.

In CIM-BPMN, a sequence diagram is created for each lowlevel BPM, which will be supported by the information system. Authors of the approach define mapping rules from low-level BPM to sequence diagram of system's external behaviour (SDESB) [17]. These rules are presented in Table III.

TABLE III

Mapping of Low-LeVel Business Process Model to Sequence DIAGRAM OF SYSTEM'S EXTERNAL BEHAVIOUR, ADAPTED FROM [17]

\begin{tabular}{|l|l|}
\hline Element in low-level BPM & Corresponding element in SDESB \\
\hline Swimlane in high-level BPM & Principal actor \\
\hline Swimlane in low-level BPM & Secondary actor \\
\hline Task performed by actor & Message sent to system \\
\hline $\begin{array}{l}\text { Task performed by the system } \\
\text { for calculation or validation }\end{array}$ & Internal message \\
\hline $\begin{array}{l}\text { Task performed by the system } \\
\text { for getting/displaying } \\
\text { information from/for actor }\end{array}$ & Response from the system to the actor \\
\hline $\begin{array}{l}\text { Flow of activities after an } \\
\text { exclusive gateway }\end{array}$ & $\begin{array}{l}\text { Successful flow is mapped to } \\
\text { actions/responses. While, other flows } \\
\text { that terminate process correctly are } \\
\text { mapped to alternative scenario, flows } \\
\text { that terminate process with errors or } \\
\text { cancelation are mapped to error } \\
\text { scenario. Both errors and alternatives } \\
\text { are represented by notes. }\end{array}$ \\
\hline $\begin{array}{l}\text { Flow that returns to previous } \\
\text { task }\end{array}$ & \begin{tabular}{l} 
A "loop" interaction operand \\
\hline Sub-process
\end{tabular} \\
\hline
\end{tabular}

Paper [17] gives an example of obtaining a sequence diagram for checkout sub-process (see Fig. 5). It is identified that the principal actor is the customer. The lower level BPM (Fig. 5) does not show any supplier secondary actors. Three internal messages are found: "validate shipping address", "check delivery mode" and "save order". The other tasks are transformed to actions/responses from/to system. The collapsed sub-process "Payment" is mapped to a "ref" interaction operand [17]. Figure 8 shows the obtained SDESB after the transformation.

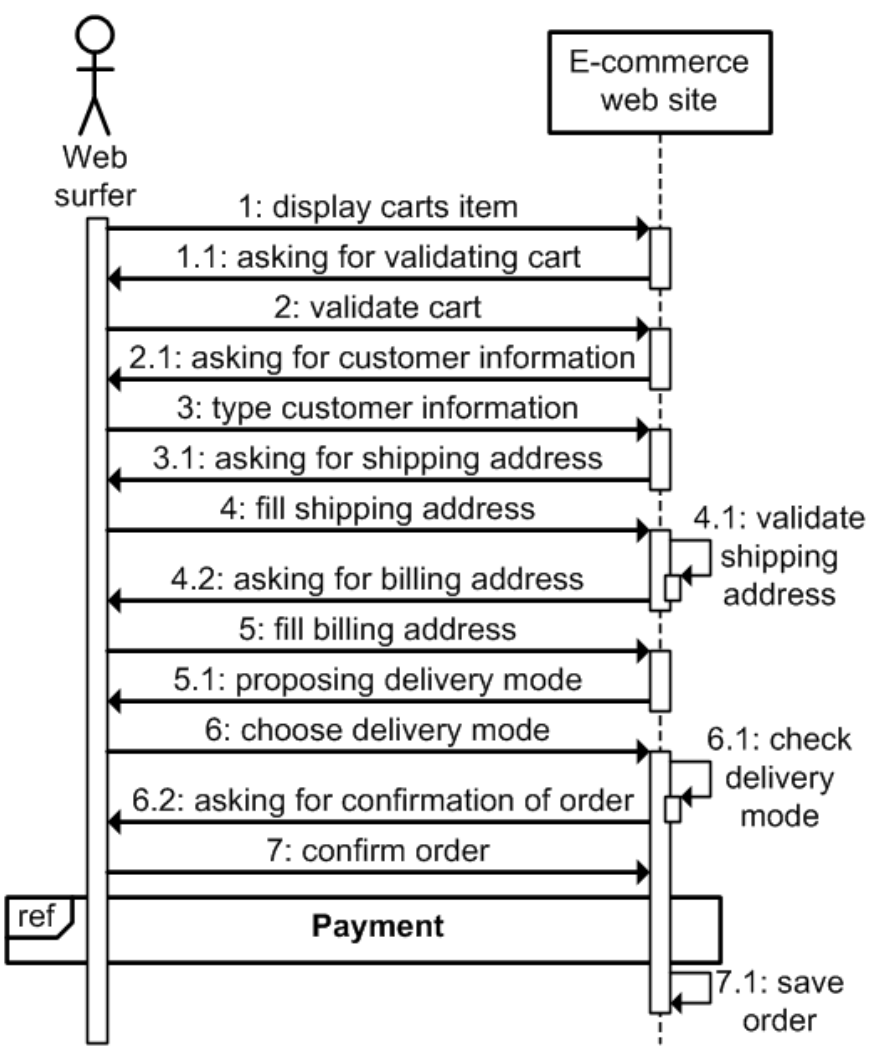

Fig. 8. Sequence diagram of system's external behaviour for the checkout subprocess, obtained by applying CIM-BPMN.

By studying the example given in Fig. 8, the author concludes that this transformation requires partial participation of the architect, i.e., coming up with appropriate text for messages.

The biggest difference between the approaches concerning the obtaining of sequence diagram is the following. In TFM4SE, a sequence diagram represents interaction between actors and the system, as well as sending messages between objects of the system. In its turn, CIM-BPMN sequence diagram shows communication between actors and the system which is presented as one object. Thus, TFM4SE sequence diagram is on lower level of abstraction. CIM-BPMN defines the transition to a low-level sequence diagram [36], but it requires additional design and description of the system's internal behaviour. The common characteristic is that both transformations require partial participation of the architect, so they are not $100 \%$ formal. However, TFM4SE also requires additional effort in coming up with names of classes and methods that will realise the functional features; and additional processing of the knowledge about the business system and the planned information system to identify system goals and corresponding functional features - this processing is directed by guidelines, but the results may vary depending on the thinking of the architect. Nevertheless, variation of the result is limited, because topology of TFM must be retained. 
TABLE IV

COMPARISON SUMMARY BETWEEN TFM4SE AND CIM-BPMN APPROACHES

\begin{tabular}{|l|l|l|}
\hline & \multicolumn{1}{|c|}{ TFM4SE (Osis et al.) } & \multicolumn{1}{|c|}{ CIM-BPMN (Bousetta et al.) } \\
\hline Business model & $\begin{array}{l}\text { TFM. } \\
\text { Formal guidelines how to get TFM from system's verbal } \\
\text { description. Comprehensive verbal description is needed. }\end{array}$ & $\begin{array}{l}\text { BPMN high-level and low-level BPMs. } \\
\text { No formal guidelines for creating the model. Comprehensive } \\
\text { verbal description is not needed, but knowledge is still required. }\end{array}$ \\
\hline $\begin{array}{l}\text { Increasing the level of } \\
\text { detail of business } \\
\text { model }\end{array}$ & $\begin{array}{l}\text { Formal operation - continuous mapping - ensures } \\
\text { consistency between abstract and detailed models. }\end{array}$ & $\begin{array}{l}\text { Expanding the sub-process is done rather intuitively which may } \\
\text { lead to inconsistency with more abstract model. }\end{array}$ \\
\hline $\begin{array}{l}\text { Transformation to } \\
\text { sequence diagram }\end{array}$ & $\begin{array}{l}\text { Model shows sending messages between objects and } \\
\text { interactions between the system and actors. } \\
\text { Coming up with names for classes and methods is required. } \\
\text { Identifying system goals and corresponding functional } \\
\text { features is required. } \\
\text { Transformation requires partial participation of the architect. }\end{array}$ & $\begin{array}{l}\text { Model shows interactions between actors and the system, but } \\
\text { does not show system's internal behaviour. } \\
\text { Transformation requires partial participation of the architect. }\end{array}$ \\
\hline
\end{tabular}

\section{CONCLUSION}

The discussion in this paper has been directed to the comparison of TFM4SE with model driven approaches that use BPMN for CIM modelling and provide CIM $\rightarrow$ PIM transformation. From the discovered ones, the author has chosen the most well-elaborated one to compare it with TFM4SE concerning CIM modelling and transformation to a sequence diagram on the PIM level. The comparison has been performed on the basis of an example (case study). The result of the research is summarised in Table IV.

As emphasised in the introduction, the main difference between TFM and BPMN model is that TFM is a formal model with mathematical background, and BPMN is not. The author believes that the improvement of the object-oriented system analysis and modelling lays in using formal methods. The author has been interested in finding out the benefits and drawbacks the formalism of TFM brings in comparison with CIM-BPMN approach. Let us start with the drawbacks. Since the comprehensive description of the system must be developed, analysis stage requires more effort than in CIMBPMN approach. Concerning transformation to PIM, the obtaining of sequence diagrams also requires more effort: the architect must come up with the names for classes and methods that will realise the corresponding functional features; also, additional analysis of knowledge about the business system and the planned information system is required to identify the system goals. On the other hand, the advantages of TFM4SE are the following. On the CIM level, the consistency between abstract and detailed models is ensured, because formal operation for manipulating the level of detail is applied. On the PIM level, the obtained sequence diagram represents inner behaviour of the information system - it shows message sending between objects. This description of inner behaviour is obtained without making any design specification, except names of classes and methods that are assigned to functional features of TFM. Only the knowledge that is obtained during the analysis phase is used.

\section{REFERENCES}

[1] J. Miller and J. Mukerji, (2003, June 12). MDA Guide Version 1.0.1. [Online]. Available: www.omg.org/cgi-bin/doc?omg/03-06-01 [February 10, 2016].

[2] J. Osis, "Software Development with Topological Model in the Framework of MDA," in Proc. of the 9th CAiSE/IFIP8.1/EUNO Int. Workshop on Evaluation of Modeling Methods in Systems Analysis and Design (EMMSAD'2004) in connection with the CAiSE'2004. vol. 1, Riga: RTU, 2004, pp. 211-220.

[3] J. Osis and E. Asnina, "Is Modeling a Treatment for the Weakness of Software Engineering?" in Model-Driven Domain Analysis and Software Development: Architectures and Functions. Hershey, New York: IGI Global, 2011, pp. 1-14. http://dx.doi.org/10.4018/978-1-61692-8742.ch001

[4] J. Osis and E. Asnina, "Is Modeling a Treatment for the Weakness of Software Engineering?" in Handbook of Research on Innovations in Systems and Software Engineering. Hershey, USA: IGI Global, 2015, pp. 411-427. ISBN 978-1-4666-6359-6. e-ISBN 978-1-4666-6360-2. http://dx.doi.org/10.4018/978-1-4666-6359-6

[5] E. Asnina and J. Osis, "Computation Independent Models: Bridging Problem and Solution Domains," in Proceedings of the 2nd International Workshop on Model-Driven Architecture and Modeling Theory-Driven Development (MDA \& MTDD 2010), in conjunction with ENASE 2010, Greece, Athens, 22-24 July, 2010. Lisbon: SciTePress, 2010, pp. 23-32. ISBN 978-9-8984-2516-4.

[6] J. Osis, E. Asnina and A. Grave, "MDA Oriented Computation Independent Modeling of the Problem Domain," in Proceedings of the 2nd International Working Conference on Evaluation of Novel Approaches to Software Engineering (ENASE 2007), Spain, Barcelona, 23-25 July, 2007. Barcelona: INSTICC Press, 2007, pp. 66-71. ISBN 978-989-8111-10-4.

[7] J. Osis, "Topological Model of System Functioning," (in Russian). Automatics and Computer Science, J. of Academia of Sciences, Riga, Latvia, no. 6, 1969, pp. 44-50.

[8] E. Asnina and V. Ovcinnikova, "Specification of Decision-making and Control Flow Branching in Topological Functioning Models of Systems," in Proc, of 10th Int, Conf, on Evaluation of Novel Approaches to Software Engineering, Spain, Barcelona, 29-30 April, 2015. Portugal: SciTePress, 2015, $\quad$ pp. 364-373. ISBN 978-989-758-100-7. http://dx.doi.org/10.5220/0005479903640373

[9] V. Ovcinnikova and E. Asnina, "The Algorithm of Transformation from UML Sequence Diagrams to the Topological Functioning Model," in Proc. of 10th Int. Conf. on Evaluation of Novel Approaches to Software Engineering, Spain, Barcelona, 29-30 April, 2015. Portugal: SciTePress, 2015, pp. 377-384. ISBN 978-989-758-100-7. http://dx.doi.org/10.5220/0005476603770384

[10] OMG (Object Management Group). "Business Process Model and Notation (BPMN), Version 2.0.2." December 2013. [On-line]. Available: www.omg.org/spec/BPMN/2.0.2/PDF [February 10, 2016].

[11] Y. Rhazali, Y. Hadi and A. Mouloudi, "Transformation Method CIM to PIM: From Business Processes Models Defined in BPMN to Use Case and Class Models Defined in UML," World Academy of Science, Engineering and Technology - International Journal of Computer, 
Electrical, Automation, Control and Information Engineering, vol. 8, no. 8, pp. 1467-1471, 2014.

[12] A.E. Fazziki et al. "A Service Oriented Information System: A Model Driven Approach," in The Eighth Int. Conf. on Signal Image Technology and Internet Based Systems (SITIS), Naples, 25-29 November, 2012. IEEE, 2012, pp. 466-473. ISBN 978-1-4673-5152-2. http://dx.doi.org/10.1109/SITIS.2012.75

[13] OMG (Object Management Group). "Service oriented architecture Modeling Language (SoaML) Specification, Version 1.0.1.” May 2012 [Online]. Available: www.omg.org/spec/SoaML/1.0.1/PDF/ [February $10,2016]$.

[14] C. Hahn, D. Panfilenko and K. Fischer, "A Model-Driven Approach to Close the Gap between Business Requirements and Agent-Based Execution," in Proc. of the 4th Workshop on Agent-based Technologies and applications for enterprise interOPerability, Toronto, Canada, 10 May 2010. AAMAS 2010, Toronto, Canada, 2010, pp. 13-24.

[15] V. D. Castro, E. Marcos and J. M. Vara, "Applying CIM-to-PIM model transformations for the service-oriented development of information systems," Information and Software Technology, vol. 53, issue 1, pp. 87-105, Jan. 2011. http://dx.doi.org/10.1016/j.infsof.2010.09.002

[16] A. Rodriguez et al. "Semi-formal transformation of secure business processes into analysis class and use case models: An MDA approach," Information and Software Technology, vol. 52, issue 9, pp. 945-971, September 2010. ISSN 0950-5849.

[17] B. Bousetta, O. El Beggar and T. Gadi, "A methodology for CIM modelling and its transformation to PIM," in Journal of Information Engineering and Applications, vol. 3, no. 2, pp. 1-21, 2013. ISSN 22245782. e-ISSN 2225-0506.

[18] W. Zhang et al., "Transformation from CIM to PIM: A Feature-Oriented Component-Based Approach," in Proc. of 8th Int. Conf., MoDELS 2005, Montego Bay, Jamaica, October 2-7, 2005. Berlin, Heidelberg: Sprienger, 2005, pp. 248-263. ISBN 978-3-540-29010-0. e-ISBN 978-3540-32057-9. http://dx.doi.org/10.1007/11557432 18

[19] J. J. Gutierrez et al., "Visualization of Use Cases through Automatically Generated Activity Diagrams," in Pro. of 11th Int. Conf., MoDELS 2008, Toulouse, France, Sept. 28 - Oct. 3, 2008. Berlin, Heidelberg Sprienger, 2008, pp. 83-96. ISBN 978-3-540-87874-2. e-ISBN 978-3540-87875-9. http://dx.doi.org/10.1007/978-3-540-87875-9 6

[20] J. Mazon, J. Pardillo and J. Trujillo, "A Model-Driven Goal-Oriented Requirement Engineering Approach for Data Warehouses," in Proc. of ER 2007 Workshops CMLSA, FP-UML, ONISW, QoIS, RIGiM, SeCoGIS Auckland, New Zealand, November 5-9, 2007. Berlin, Heidelberg: Sprienger, 2007, pp. 255-264. ISBN 978-3-540-76291-1, e-ISBN 978-3540-76292-8. http://dx.doi.org/10.1007/978-3-540-76292-8 31

[21] E. Yu, "Modelling Strategic Relationships for Process Reengineering." Ph.D. thesis, University of Toronto, Canada, 1995.

[22] S. Kherraf, E. Lefebvre and W. Suryn, "Transformation from CIM to PIM Using Patterns and Archetypes," in 19th Australian Conf. on Software Engineering, ASWEC 2008, Perth, Australia, 26-28 March 2008. IEEE 2008, pp. 338-346. ISBN 978-0-7695-3100-7. ISSN 1530-0803. http://dx.doi.org/10.1109/ASWEC.2008.4483222

[23] M. Kardos and M. Drozdova, "Analytical method of CIM to PIM transformation in Model Driven Architecture (MDA)," J. of Information and Organizational Sciences (JIOS), vol. 34, issue 1, pp. 89-99, 2010. ISSN 1846-3312. e-ISSN 1846-9418.

[24] E. Asnina and J. Osis, "Topological Functioning Model as a CIMBusiness Model," in Model-Driven Domain Analysis and Software Development: Architectures and Functions. Hershey, New York: IGI Global, 2011, pp. 40-64. http://dx.doi.org/10.4018/978-1-61692-8742.ch003

[25] J. Osis and A. Slihte, "Transforming Textual Use Cases to a Computation Independent Model," in Model-Driven Architecture and Modeling Theory-Driven Development: Proc. of the 2nd Int. Workshop (MDA \& MTDD 2010), Greece, Athens, 22-24 July, 2010. Lisbon SciTePress, 2010, pp. 33-42. ISBN 978-9-8984-2516-4.

[26] A. Slihte, J. Osis and U. Donins, "Knowledge Integration for Domain Modeling," in Proc. of the 3rd Int. Workshop on Model-Driven Architecture and Modeling-Driven Software Development (MDA \& MDSD 2011), China, Beijing, 8-11 June, 2011. Lisbon: SciTePress, 2011, pp. 46-56. ISBN 978-9-8984-2559-1.

[27] A. Slihte et al., "Advancements of the Topological Functioning Model for Model Driven Architecture Approach," in Proc. of the 3rd Int Workshop on Model-Driven Architecture and Modeling-Driven Software Development (MDA \& MDSD 2011), China, Beijing, 7-11 June, 2011 Lisbon: SciTePress, 2011, pp. 91-100. ISBN 978-9-8984-2559-1.
[28] J. Osis and E. Asnina, "Derivation of Use Cases from the Topological Computation Independent Business Model," in Model-Driven Domain Analysis and Software Development: Architectures and Functions. Hershey, New York: IGI Global, 2011, pp. 65-89. http://dx.doi.org/10.4018/978-1-61692-874-2.ch004

[29] OMG (Object Management Group). "OMG Unified Modeling Language (OMG UML), Version 2.5.” March 2015. [Online]. Available: www.omg.org/spec/UML/2.5/PDF [February 10, 2016].

[30] J. Osis et al., "Dependencies among Architectural Views Got from Software Requirements Based on a Formal Model," Applied Computer Systems, vol. 16, pp.5-12, 2014. ISSN 2255-8683. e-ISSN 2255-8691. http://dx.doi.org/10.1515/acss-2014-0007

[31] J. Osis, E. Asnina, and A. Grave, "Formal Problem Domain Modeling within MDA," in Communications in Computer and Information Science (CCIS). Software and Data Technologies: Second Int. Conf. ICSOFT/ENASE 2007: Revised Selected Papers, Germany, Berlin, 22-25 July, 2007. Berlin: Springer-Verlag Berlin Heidelberg, 2008, pp. 387-398. ISBN 978-3-5408-8654-9. e-ISBN 978-3-5408-8655-6. ISSN 1865-0929.

[32] J. Osis, E. Asnina, and A. Grave, "Computation Independent Representation of the Problem Domain in MDA." e-Informatica Software Engineering Journal, vol. 2, issue 1, pp. 29-46, 2008. ISSN 1897-7979.

[33] E. Asnina, "Backward Requirements Traceability within the Topologybased Model Driven Software Development," in Proc. of the 3rd Int. Workshop on Model-Driven Architecture and Modeling-Driven Software Development (MDA \& MDSD 2011), China, Beijing, 7-11 June, 2011. Lisbon: SciTePress, 2011, pp. 36-45. ISBN 978-9-8984-2559-1.

[34] O. El Beggar, B. Bousetta and T. Gadi, "Generating methods signatures from transition state diagram: A model transformation approach," in Information Science and Technology (CIST), 22-24 October, 2012. 2012, pp. 4-9. http://dx.doi.org/10.1109/CIST.2012.6388054

[35] O. El Beggar, B. Bousetta and T. Gadi, "Automatic code generation by model transformation from sequence diagram of system's internal behavior." Int. J. of Computer and Information Technology (IJCIT), vol. 1, issue 2, pp. 129-146, November 2012. ISSN: 2279-0764.

[36] B. Bousetta, O. El Beggar and T. Gadi, "Automating Software Development Process: Analysis-PIMs to Design-PIM Model Transformation," Int. J. of Software Engineering and Its Applications, vol. 7, no. 5, SERSC, pp. 167-196, 2013. ISSN 1738-9984.

[37] B. Bousetta, O. El Beggar and T. Gadi, "Generating operations specification from domain class diagram using transition state diagram," Int. J. of Computer and Information Technology (IJCIT), vol. 2, issue 1, pp. 29-36, January 2013. ISSN: 2279-0764.

[38] K. Fernandez Cespedes, J. Osis and G. Alksnis, "Lessons Learned by using the Integrated Domain Modeling Toolset." in Proc. of the 10th Int. Conf. on Evaluation of Novel Approaches to Software Engineering, ENASE 2015, Spain, Barcelona, 28-30 April, 2015. Portugal:SciTePress, 2015, pp. 352-363. http://dx.doi.org/10.5220/0005477703520363

[39] J. Osis and E. Asnina, "Topological Modeling for Model-Driven Domain Analysis and Software Development: Functions and Architectures," in Model-Driven Domain Analysis and Software Development: Architectures and Functions, Hershey, New York: IGI Global, 2011, pp. 15-39. http://dx.doi.org/10.4018/978-1-61692-874-2.ch002

[40] U. Donins and J. Osis, "Topological Modeling for Enterprise Data Synchronization System: A Case Study of Topological Model-Driven Software Development," in Proc. of the 13th Int. Conf. on Enterprise Information Systems, vol. 3, China, Beijing, 8-11 June, 2011. Beijing: SciTePress, 2011, pp.87-96. ISBN 978-9-8984-2555-3.

[41] J. Osis and U. Donins, "Formalization of the UML Class Diagrams," in Evaluation of Novel Approaches to Software Engineering: 3rd and 4th Int. Conf., ENASE 2008/2009: Revised Selected Papers, Italy, Milan, 9 10 May, 2009. Berlin: Springer-Verlag, 2010, pp. 180-192. ISBN 9783-6421-4818-7. e-ISBN 978-3-6421-4819-4. ISSN 1865-0929. http://dx.doi.org/10.1007/978-3-642-14819-4_13

Arturs Solomencevs is currently Master's student and Research Assistant at the Department of Applied Computer Science, Riga Technical University. He actively participates in the scientific research project called Topological Functioning Model for Software Engineering (TFM4SE)

E-mail: Arturs.Solomencevs@gmail.com 